\title{
Huge Intracranial Meningioma Mimicking Alzheimer Dementia
}

\author{
Bohm Choi, San Jung, Sung-Hee Hwang, Yang-Ki Min, Chae Young Lee, Song Hwangbo, Inyoung Choi \\ Department of Neurology, Hallym University Kangnam Sacred Heart Hospital, Hallym University College of Medicine, Seoul, Korea
}

Corresponding Author:

San Jung, MD

Department of Neurology, Hallym University Kangnam Sacred Heart Hospital, Hallym University College of Medicine, 1

Singil-ro, Yeongdeungpo-gu, Seoul 07441, Korea

Tel: +82-2-829-5125

Fax: +82-2-847-1617

E-mail: neurojs@hallym.ac.kr

Received: March 9, 2017

Revised: June 8, 2017

Accepted: June 12, 2017
Secondary dementia can present as a slow progression of clinical symptoms that mimic Alzheimer dementia. We report on a 73-year-old female presenting with slow, progressive memory impairment, caused by intracranial tumors, which mimicked Alzheimer dementia. A neurological examination revealed no focal deficit, but an extensive neuropsychological evaluation showed a decrease in attention and language, as well as in visuospatial, memory and executive functions, which typically present in Alzheimer dementia. Magnetic resonance imaging revealed 2 large brain tumors: a midline meningioma on the anterior frontal fossa and a right latero-temporal bone tumor. Investigative imaging was essential for diagnosis.

Key Words: Alzheimer dementia, Meningioma, Dementia

\section{INTRODUCTION}

Dementia refers to the condition in which social or occupational dysfunction is experienced, due to impairment or loss of cognitive function, originating from organic brain damage. Various disease groups can cause dementia symptoms, including Alzheimer dementia, which is the most common form of the condition. People with Alzheimer dementia experience slow, progressive memory decline and difficulty in communication, followed by a further deterioration of their other cognitive abilities. As the disease progresses, behavioral and psychological symptoms become apparent, and eventually, everyday activities become less frequently undertaken ${ }^{1)}$. Major symptoms include memory disorder, along with verbal, visuospatial, and executive function disorder. Alzheimer dementia is diagnosed using 2 evaluation methods: history taking and noting a change in cognitive function on neuropsychological testing ${ }^{2,3)}$. Recently, diagnostic criteria using cerebrospinal fluid (CSF) biomarkers and diagnostic tools, such as magnetic resonance imaging (MRI), fludeoxyglucose-positron emission tomography (PET) and amyloid PET scans have been employed, in addition to the characteristic clinical progress of Alzheimer dementia, such as episodic amnesia ${ }^{4)}$.

This case study reports on a patient initially misdiagnosed with Alzheimer dementia, who presented with slowly declining cognitive function, but was ultimately diagnosed, using MRI, as having multiple intracranial tumors, including meningioma.

\section{CASE REPORT}

A 73-year-old female patient visited our memory clinic presenting with memory decline that had started 3 years earlier. The patient was right-handed and her education level was middle school graduate. Approximately 3 years beforehand, several symptoms first appeared. These included having difficulty recalling exactly what she heard and said, and consequently her responses might be relevant to a conversation or could be unrelated to it. In addition, she had called herself by a different name and had not been able to accurately recall the names of previously familiar objects. She used demonstrative nouns instead of accurate names. She could not understand a complex sentence and her speaking fluency declined. She started to display clumsiness while using cooking utensils, for example, forgetting how to use a ladle or a blender. One year prior to her visit, she became unable to recall her home address and forgot appointments or schedules more frequently. Then, 6 months previously, she could not recall whether she had eaten, and denied eating even when she had. Her caregiver helped with taking her medication because she frequently forgot to take it at the appropriate times. About 2 years prior to her visit, there had been 
several occasions where she had become unsure of her way while trying to come home when travelling by bus from a suburb to the bus stop at her own town. She had become indifferent to housework and to personal hygiene. Her caregivers frequently noted that she sat motionless in front of the television. She had also developed a tendency to overeat. Her medical history indicated that she had received treatment for diabetes, hyperlipidemia, and hyperthyroidism at a local clinic.

A physical examination conducted at the time of the visit revealed no specific findings, and a neurological test showed alert consciousness, while motor and sensory function tests, deep tendon reflexes, cerebellum function testing, and gait analysis were within the normal range. To determine an accurate neuropsychological assessment of the patient, a Seoul Neuropsychological Screening Battery test was conducted. Using the modified Korean Mini-Mental State Evaluation test, the results were scored as follows: time orientation, 1 out of 5 ; place orientation, 3 out of 5 ; memory registration, 3 out of 3 ; attention and calculation, 1 out of 5 ; memory retrieval, 0 out of 3 ; verbal ability, 7 out of 8 , and; visuospatial ability, 1 out of 1 ; resulting in a total score of 16 , which indicated an apparent decline of cognitive function. Keeping alert, in respect of attention function, was less than normally expected. Among the language-related functions, reading and writing levels remained similar to her prediagnosed state, but a Korean Boston Naming Test gave results of 13 out of 60 , indicating a clear decline. The patient was unable to demonstrate how to use a spoon or chopsticks and failed a calculation test. For visuospatial assessment, a Rey Complex Figure Test showed a decrease to $1 \%$ or less. After assessing learning and memory functioning, using a Seoul Verbal Learning Test, results indicated that immediate recall, delayed recall, and recognition had all declined to $1 \%$ or less. Repetitive learning was unsuccessfully undertaken, while nonlinguistic testing using the Rey Complex Figure Test showed
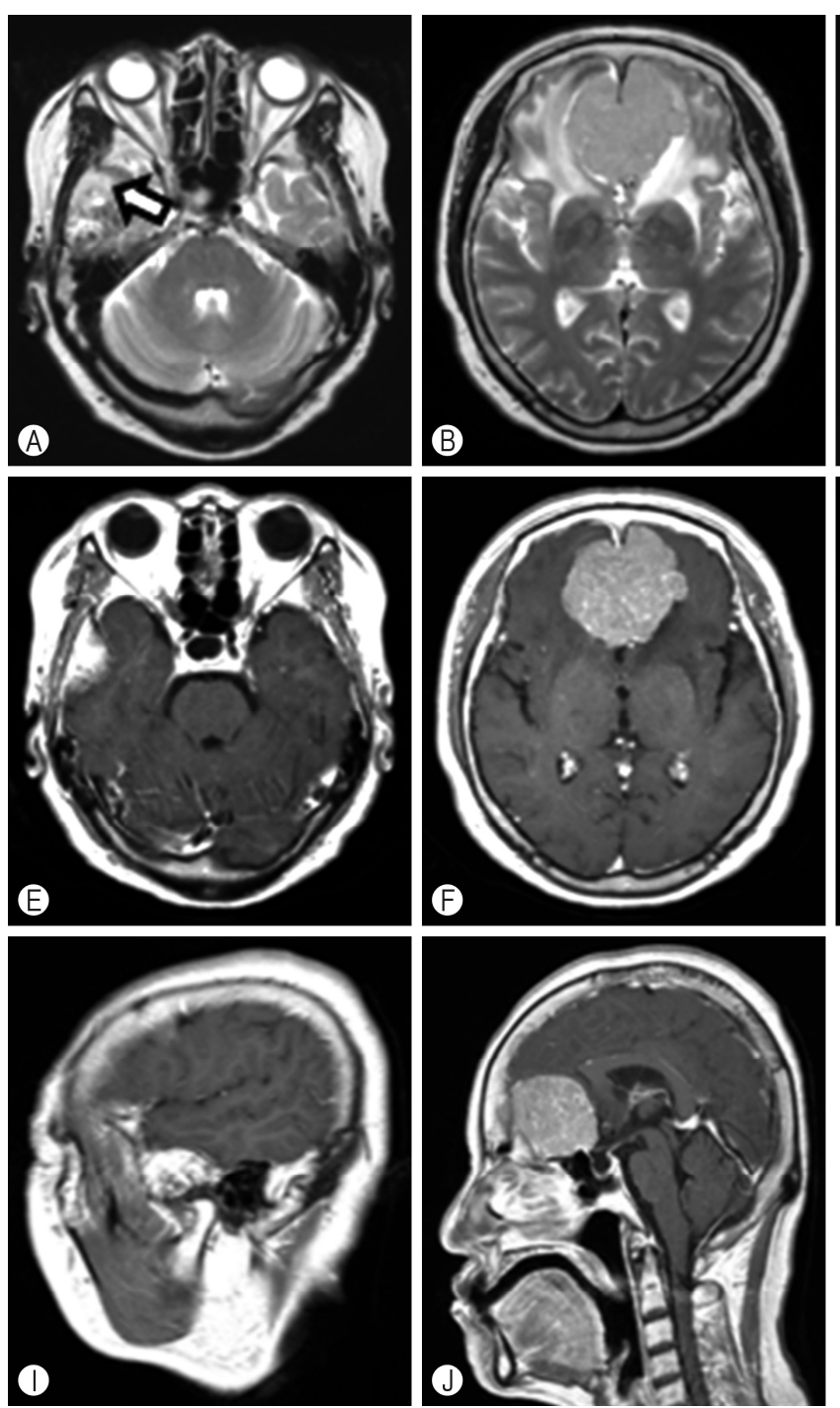
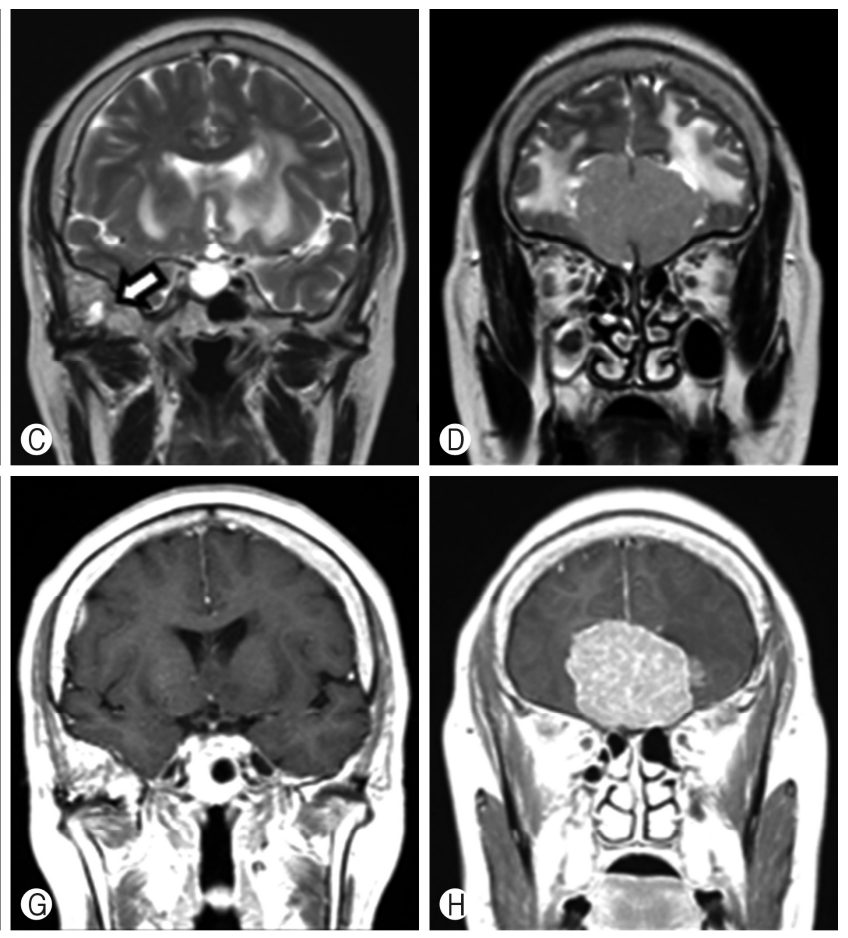

Fig. 1. Results of brain magnetic resonance (MR) imaging T2 weighted MR images of axial and coronal view (A-D) and postenhancement T1 weighted MR images of axial, coronal, and sagittal view (E-J) show well enhanced midline tumor on anterior fossa meningioma $(\mathrm{B}, \mathrm{D}, \mathrm{F}, \mathrm{H}, \mathrm{J})$ and right temporal bone tumor (cavernous hemangioma) (A, C, E, G, I). 
that instant and delayed recall were not possible, and a recognition test result gave a result of $1 \%$ or less, all indicating a low score. Frontal and executive functioning did not show evidence of motor impersistence, but decreased abilities for sequential motor programming and motor coordination were observed in the Fist-Edge-Palm Test and in the alternating hand movement test. Psychomotor slowing was observed in the Digit Symbol Coding test. Generative naming function decreased in the Controlled Oral Word Association Test, and there was no inhibitory control noted in the Luria Loop Test. A diagnosis of depression was ruled out, as evidenced by a score of 6 in the Short form Geriatric Depression Scale. These results overall suggested severe dysfunction in linguistic memory, visual memory, registration, and attention ability. In linguistic ability, there was a decreased ability to understand complex sentences and name objects. Ideo- motor apraxia and dyscalculia were observed, visuospatial function was decreased, and frontal and executive functions were severely impaired, except for motor impersistence. This result corresponded to frontal, temporal, and parietal cortex dysfunction.

A T2-weighted head and neck MRI revealed a 5- $\mathrm{cm}$ mass surrounded by edema at the anterior fossa, attached to the intracranial falx and surface of the dura mater, with thickening of the right temporal bone, detected as irregular high-resonance. The mass attached to the intracranial dura mater was enhanced in a T1-enhanced image with contrast, and believed to be a meningioma. The patient was then referred to the Neurosurgery Department (Fig. 1)

\section{DISCUSSION}

The National Institute on Aging and the Alzheimer's Association proposed revising the criteria for diagnosing Alzheimer disease in 2011. According to these criteria, the disease can be diagnosed when a person has difficulty maintaining his or her daily activities, and having two or more symptoms arising within the following categories: cognitive function, executive function, visuospatial function, language function, and personality change. Memory impairment has been eliminated as a criterion requirement. An additional component in the Alzheimer's dementia revised criteria has been the incorporation of biomarkers to increase the certainty of diagnosis").

Besides Alzheimer dementia, various diseases can cause dementia, and to avoid missing treatable causes, clinicians should identify relevant past medical history, social history, and medication history factors, as well as conduct neurological examinations including tests for vision and hearing impairment. In this report, the patient was clinically assessed as having Alzheimer dementia, as evidenced by slowly progressive changes in cognitive function, executive function and visuospatial function beginning 3 years prior to her visit to our memory clinic. Furthermore, she had frontal lobe dysfunction. She did not show any neurologically abnormal symptoms, such as headache, seizure, gait disturbance or movement symptoms like dystonia or tremor. According to previous studies, approximately 50\% of meningioma cases at the olfactory groove can show anosmia, but our patient did not present with anosmia ${ }^{5)}$. On the other hand, the neuropsychological tests showed a marked deterioration in the global cognition function including memory. On the brain MRI, a 5-cm meningioma surrounded by edema was identified, which was putting pressure on bilateral medial frontal lobe tissue on the midline of the anterior brain fossa. At the same time, a cavernous hemangioma attached to the temporal lobe was found, which was also putting pressure on the right temporal lobe. These tumors showed distinct enhancement with T1-weighted images, using contrast. As CSF biomarkers (a beta amyloid decrease and a total tau or phosphorylated tau protein increase) were not used and amyloid PET was not performed, we could not identify whether Alzheimer dementia was also present. However, on MRI, outstanding hippocampal atrophy or global brain atrophy was not observed.

The clinical features of meningioma can be diverse, and most are asymptomatic. As an early symptom of meningioma, decline in cognitive function is less common. In previous records by Mr. Wylie McKissock at the National Hospital and Mr. Valentine Logue at Atkinson Morley Hospital, Only $3.4 \%$ of meningioma cases showed dementia as the initial symptom preceding the development of those commonly regarded as indicative of cerebral tumor ${ }^{5}$.

Meningioma at the olfactory groove is characterized by its rareness and by a gradual growth, which explains why diagnosis is more likely to occur when the tumor is larger. The patient we presented took approximately 3 years to be accurately diagnosed. Differing from untreatable Alzheimer dementia, most dementia symptoms caused by meningioma can be reversed with surgical treatment.

In conclusion, the authors have reported one case of coexisting extensive meningioma at the midline olfactory groove and a cavernous hemangioma compressing the right temporal lobe, which was once misdiagnosed as Alzheimer dementia, since the patient did not present with other neurological abnormal symptoms.

Conflicts of Interest Disclosures: The researchers claim no conflicts of interest.

\section{REFERENCES}

1. Ryu SY, Lee SB, Kim TW, Song IU, Oh EY, Choi HY. Relationship between neuropsychiatric symptoms and activities of daily living in Alzheimer's disease. J Korean Geriatr Soc 2010;14:131-8. 
2. Hyman BT, Phelps CH, Beach TG, Bigio EH, Cairns NJ, Carrillo $\mathrm{MC}$, et al. National Institute on Aging-Alzheimer's Association guidelines for the neuropathologic assessment of Alzheimer's disease. Alzheimers Dement 2012;8:1-13.

3. McKhann G, Drachman D, Folstein M, Katzman R, Price D, Stadlan EM. Clinical diagnosis of Alzheimer's disease: report of the NINCDS-ADRDA Work Group under the auspices of Depart- ment of Health and Human Services Task Force on Alzheimer's Disease. Neurology 1984;34:939-44.

4. O'Mahony D, Walsh JB, Coakley D. 'Pseudo-Alzheimer's' and primary brain tumour. Postgrad Med J 1992;68:673-6.

5. Sachs E Jr. Meningiomas with dementia as the first and presenting feature. J Ment Sci 1950;96:998-1007. 